\title{
A Survey on Evolution of Cognitive Robotics with Internet of Things
}

\author{
Syed Umar ${ }^{1}$, Endal Kachew Mosisa ${ }^{2}$
}

${ }^{1}$ Professor, Department of Computer Science, Wollega University, Nekemte, Ethiopia

${ }^{2}$ Dean \& Assistant Professor, Department of Mechanical Engineering, Wollega University, Nekemte, Ethiopia

\begin{abstract}
Article Info

Volume 8 Issue 2

Page Number: 337-344

Publication Issue :

March-April-2021

Article History

Accepted : 04 April 2021

Published : 20 April 2021

The Internet of Things (IoT) is shifting across various fields, specifically the Autonomous Internet of Mobile Things (IoMT), Autonomous Internet of Things (A-iOT), Autonomous System of Things (ASoAT), Internet (ASoT), and the Autonomous System of Things (ASoT) as well as to reflect/embr/Use the Internet of IoT in Internet of Mobile Things (IoMT), Autonomous Internet of Things (A-IoT), Autonomous (A-iTC) as well as in Internet of Mobile Things (IoMT) and Autonomous system (AS), melding context-based network technology and complex objects with smooth plat-forms and app incorporation, as critical IoT advancements, presenting new network creation and expansion threats, and implementation problems, and general sensor/ limitations, and sensors and actuators, are several of the new development and operational issues that also pose a new challenges, it requires to handle The expansion of interregional rivalry represents fresh interregional problems. Because parallel processing and concurrency in the Internet of Things need different approaches to accommodate intelligent "components,", we are currently have to devise new ways to integrate collaborative robots (COBOTS) "cognitive devices" and/on-service environments will rapidly add additional services, sustain, and change, and becoming part of IoT networks. For both of these reasons, resource aspects, self-healing, self-repairing, and context-based/service compositions of cognitive resources are crucial for application development and network incorporation. to include: This chapter is written to provide a concise summary of the IoRT philosophy, technology, and possible problems as well as their various advances and implementations so that the reader can get a sense of their potential outcomes.
\end{abstract}

Keywords : IoRT, COBOTS, A-iOT, ASoT, IoMT

\section{INTRODUCTION}

Together, artificial intelligence (AI), automation, machine learning, and the intelligence of several cooperating machines will be the latest in the evolution of the Internet of Things. Robotics is used in machines that are intended to do work intensive and repetitive work, such as factory 
robots, to support decision- and learning-based decision making and learning algorithms instead. This union of these various disciplines lays the groundwork for programmable machines, integrating robotics with machine learning, lays the groundwork for robotic machines to be created.

As they progress to the next level of intelligent behaviour, machine learning, algorithms and software that are non-specific use (i.e. they don't need a parametrically prescribe a response) utilize statistics, which may be referred to as statistical pattern detection, statistical parametric algorithms, recommendation systems and swarms both begin to function autonomously. It is part of the Internet of Things (IoT), where computer machines, intermediate units, and networks extend their activities by communicating with their immediate environments to gather data [and further communicate with] This field is where we can expect to see all intelligent functions and IoT data centers use-allocation technologies.

The usage of communication-constrained robots are finding ways to increase their accessibility, use of sensors and other network tools is another emerging and converging concept in robotics. linked robots are part of a "networked systems," a term that means robots that the user controls remotely by way of the internet or LAN The network may be wired or wireless, it would be able to use various protocols such as TCP, UDP, 802.11, or other protocols, such as cellular wireless. The wide variety of emerging technologies being introduced covers a variety of topics, such as robotics and discovery. Two classes of networked robots have been defined by the IEEE Society of Robotics and Automation's Technical Committee on Networked Robots [10].

supervised robot control systems, through which a human supervisor sends instructions and receives information through the network to students, researchers, and the public are invaluable to all purposes since they provide plentiful services to students and the science community as much as they make them available to the public.

robot systems, where no on-human interaction is required and robots share data with each other over the network Instead of moving the sensors from one end of the territory to the other, the network may be extended, increasing the radius of their influence, thus enabling the robots to collaborate in the same territory. the robots will also manage, deploy, patch, and replace the sensor network for durability, which increases the network's functionality.

With the two major subclasses of networked robots, programming security and improving operations go hand in hand, as well as short and extended terms, upgrading, are needed.

More powerful computers and those with little or local processing (e.g., globally consistent maps) are needed for connected robots, multiple robots. The functionality offered by this network expands activities including teaming which tele-operation, and allows on-the- fly and on-the-reprogramming of robots to take place in real time [tele-operation includes teaming up with other robots and on- this links these networks allows for in-the-the-fly human robot contact and reprogramming of the robots for fast response]. Although previously we have seen the use of these technologies in facilities, they have recently found their way into the customer sector to assist in tele-presence and health care meetings over the internet. With the addition of cloud-based robotic systems, scalable capabilities are now extendable to meet cloud infrastructure scene and increased elasticity of robotic architecture helps make cloudbased robot-to-to-robot communication possible, allowing robots to function even though one is having difficulties and emulation is one of the issues, and cloud robotics has the ability to use mutual expertise. 
The fusion of advanced sensing/actuating, networking, local and remote computing, carry the original vision for the IoT to a wholly different stage, and one that provides whole new groups of possibilities for IoT and robotics solution vendors, as well as consumers of their devices. The definition enables baseline characteristics [1] that can be summarized as follow:

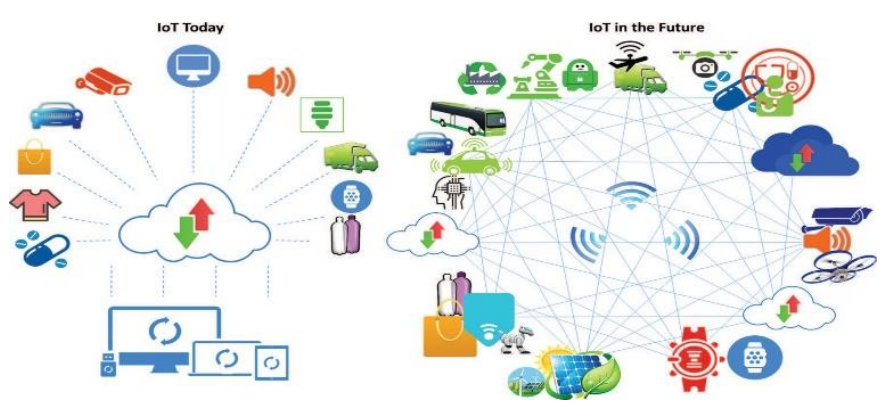

Figure 1. From a centralized cloud to distributed edge IoT platforms and applications.

- Define and describe the characteristics of robotics technologies that distinguish them as a separate, unique class of IoT objects, and one that differs considerably from the common understanding of IoT edge nodes as simple, passive devices.

- Reveal how the key features of robotics technology, namely movement, mobility, manipulation, intelligence and autonomy, are enhanced by the IoT paradigm, and how, in turn the IoT is augmented by robotic "objects" as "intelligent" edge devices.

- Illustrate how IoT and robotics technologies combine to provide for ambient sensing, ambient intelligence and ambient localization, which can be utilized by new classes of applications to deliver value.

IoT, cognitive computing and artificial intelligence technologies integration is part of the new developments foreseen for IoT applications in various smart environments.

\section{RELATED WORK}

A. Gluhak, O. Vermesan et el. [5] provides a methodology to help us understand what IoT platforms have to offer and what we need to do to stay aware of in order to thrive in the room. using the suggested approach, IoT platforms would be able to recognise their current role in the IoT ecosystem and improve on their weak points to help their organization build a better IoT ecosystem In preparation for guiding the expansion of the IoT networks and to become established and impactful in society, the surrounding innovation, the paper provides the necessary guidelines to help identify goals and build effective ecosystems. The final result of the study will be published with the publication of the different findings identified as the usage and application papers to be planned for IoT later this year.

J. Wan, S. Tang, H. Yan et el. [7] discusses the various aspects of cloud robotics and divides it into root and growth of cloud computing, followed by detailed analysis of the communications and design of cloud computing. Robot Clouds are discussed in this essay, as well as the main technology that will be critical to driving their growth are enumerated. Any facets of cloud robotics framework concepts are addressed in more detail in the final section of this document. From a multi-robot network to a multi-wide application of computational tools, cloud robotics facilitates systems and expands learning to unprecedented new heights. simplacing higher computing demands on the cloud platforms allows multirobot systems to run simple algorithms without high-performance onboard facilities, reducing overall hardware costs by orders of many fold. Ermac indicated the big data-supported cloud robotic service, or cloud robotics for greater mobility and mapping precision. They advocated the development of cloud robotics for big data, cloud computing, cloud robotics, and other areas.

L. Riazuelo et al. [8] discussed about one or any robot in the same ecosystem can gain from experience 
already preserved by other robots in that specific environment that have already extended is: Thanks to the Robo Earth method, new robots will retrieve the information. The proposed method describes and determines the semantic relationships between different arti facts thus taking into account a map representation of the existing information. using this approach, it is possible to evaluate information dependent reasoning on entity relationships To put it another way, we have seen that designing and deploying this mapping framework can be done in the cloud and using network and cloud-based services We do not need to include an update, but the robot does need to provide a situational [robot description] earth; we will follow the order sent by SRDL and complete the task automatically. If the operation has been done, the action's outcome is preserved in the database for potential future usage by other robots is also. Pioneering of a web-enabled cloud semantic Cloud semantic mapping technology that has already been demonstrated in the example of map and search has now been thoroughly demonstrated by our novel map-as-based object focused method.

G. Hu, W. P. Tay et el. [9] A cloud-enabled robot computing architecture allows one to use many robots and expand computational and information-sharing capabilities. cloud-robot design relies on machine-on on ad hoc cloud created by machine-to-machine (M2M) communications and the machine-to-machine (M2M) network to perform. Cloud robotics uses an elastic computing, in which resources are made available in the cloud and then distributed among shared resources to run various tasks, which frees up computing resources for use in applications and also enables robotic offloading and data sharing. We develop and examine various types of connectivity protocols, along with many elastic computing models for certain specific types of applications. With regard to computing, we address the difficulties of networking, as well as communication and defense, we introduce cloud-robotics as an interesting field of future study.

\section{METHODOLOGY}

The rise of machine learning in the Internet of Things (IoT) is gradually evolving to include use cases across different verticals and leading to defining the contours of a continually-evolving need for distributed machine learning. such a requirement is partly due to the large quantities of complex and noisy data that the IoRT produces and the fact that it has a wide application scope It's predicted that in the long term, the integration of IoT and machine learning would allow for flexible and intelligent IoT technologies to respond to new environments thus allowing higher degrees of personalization and use for ML apps and service management and services. Figure 4 summarizes how a distributed learning relates to a robotic stuff, showcasing computational, sensing, and actu-driven components (depicted by different colours and sizes in the figure). Fig. 4 . Below are the rules for sizing the model to use on the computing system it runs on: There may be versions that only need to provide feedback to be provided with data for use on a remote device. Furthermore, for instance, cloud services, a. a greater number of more complicated learning model, including hierarchical or quad-tree or some other wide classifier models, may be used to make aggregated predictions from many narrower and distributed models, for example, models (e.g. at the level of regional gateways).

Although interface-specific access prediction information is a critical to successful implementation of software and services in the learning system, various access protocols must be implemented to learn such predictions. A critical feature a provider must be able to include is the ability to automatically allocate new predictive assignments based on data provided by the IoRT components as the user requests 
them, and implementation of related learning modules to gain practical expertise.

The MLS (Managed Links Service Content Services) service must not only be provided even at the outermost nodes of the IoRT (Internet of Services), particularly in terms of geographic expansion. Raw data will be delivered close to the end-users and used to warn them about forecasts as quickly as possible, and then this dispersed and embedded intelligence will be able to do early data fusion and quantitative analyses on that to further drive decisions to make them understandable at a higher level. It which aggregate the sensed data and pass that data along to additional nodes, building intelligent models that make incremental inferences about that aggregate, each of which has the capability to use their own network and to calculate predictions and processes that could potentially resulting in the construction of intelligent network models, creating various applications that take information as inputs. such interfaces serve to bring about a picture of the function-related challenges, on the one hand, that helps limit the task's complexity on the one hand, and spread the system entities across the system (depicted in Figure 2) as well as a concept of the system.

The primary task is to allow distributed machine learning in the IoT (that is, different types of datacapable systems can communicate and learn as they develop self-service applications or smart apps that evolve alongside).

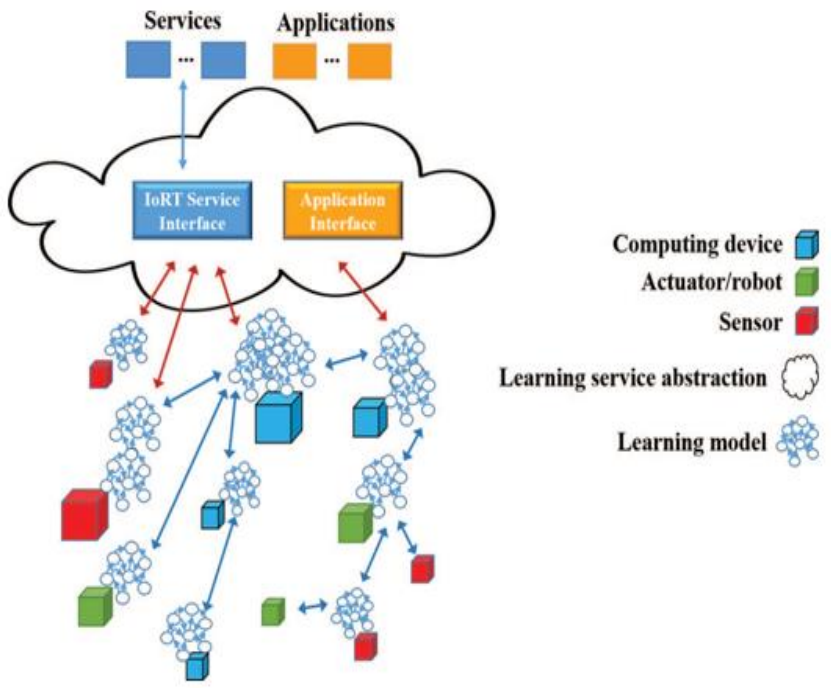

Fig 2: Architecture of an IoRT learning system highlighting the distributed nature of the service and the thing-embedded learning models.

Extending application and service capabilities involves solving critical problems about how apps should figure out how information can be processed today and the particulars of existing items can be included in these so that we can relate to future plans. Nowhere in which learning module do you want to deploy the module?" the matter of fact that the use of resources matters to choose a trained learning module and keep track of that; and in regards to its consumable resource use, making sure to keep an eye on supply and refill when necessary (e.g. to dynamically transfer a predictor if resources are insufficient or critical).

\section{Robotics and IoT Multi Annual RoadMap}

The concerned author is directed to the SRUI member is referred to the path for more in depth on future Prime opportunities for robotic technology, and is put on the pathway to the IoT road map (2020 Robotics Multi-Annual Route)." A roadmap that serves as a strategic reference for robotics researchers in advancing and analyzing their respective projects, long-in-coming innovation ideas and ideas and milestones to Robotic group members that show progression throughout the medium term. 
New concepts like Internet of Things (IoT) and cyberphysical systems promise to change the environment of innovation with regards to making large effects on fields like cyber security, transportation, healthcare, logistics, and city management, for example

- If changes in the ease of working between farm machinery and outside of the field, as well as communications between the machines improve farm and their capability expand, so does the capacity to move crops more efficiently and productivity on the farm itself.

- Because robotics still offers useful and innovative services to both government and the private industries, it would likely see increased use in these domains. The various types of infrastructure include for infrastructure for law enforcement and the ordinary systems also include, but are not limited to, ports, water pipes, sanitation facilities, sewage plants, and trash disposal.

- The future extension of multi-modal data generated by robotics technology enables certain systems to be created and implemented more rapidly and assets to be enhanced that use this data to perform multi-functionality.

- All in all, unsafe, dirty and hostile conditions and the obligation to keep them clean enable robots to possess an extended inspection, repair, maintenance, and cleaning range The relevant technological improvements involve using helicopters, such as wind turbines or highpowered transmission lines for sensors that can be installed on hard-to-reach locations, such as towers or substations.

- Within logistics and transport facilities, robotics manage collecting products, sorting, and stocking, as well as delivery, delivery, and storage, and unloading, e. There are also valuable applications where robots like selecting and packing centers, delivery stations, warehouses, freight depots, and transportation hubs, including the last mile, and all distribution points of communication with consumers.

\section{CONCLUSION}

With the first revolution (from the personal computers, to the telephone, to mobile devices like smart phones and wearable devices, to the internet of things) has dramatically improved the handling info, the personal robot would allow a qualitatively similar step in the physical world. A key component of the potential deployment of Internet of Things and artificial intelligence would be to give robotics a major position in the company's service. These attributes often enable IoT to be used for several domains: re- individual functionality for reconnect with individuals, such as applications, computers, and people interactions, allowing it to better fit numerous use cases. This is an expansion of robotics and artificial intelligence together with Internet of Things (IoT) that enables robots to execute tasks more capably and often cooperates with human beings. Because of the advent of IoT platforms, numerous robots may easily be interconnected with both themselves and with objects, thereby enabling contact with the two-parties' capacity to move data without human intervention Robotic process ability obtained which outcomes such as increased cognitive capacities to further extend the reach of machine learning while also enhances device reliability and responsiveness and dependability while mitigating threats to consumers as well as well as assisting with human and machine-robot interaction and complex physical and behavioural human-robot cooperation.

\section{REFERENCES}

[1]. O. Vermesan and P. Friess (Eds.). Digitizing the Industry Internet of Things Connecting the Physical, Digital and Virtual Worlds, ISBN: 
978-87-93379-81-7, River Publishers, Gistrup, 2016.

[2]. O. Vermesan and P. Friess (Eds.). Building the Hyper connected Society - IoT Research and Innovation Value Chains, Ecosystems and Markets, ISBN: 978-87-93237-99-5, River Publishers, Gistrup, 2015.

[3]. O. Vermesan, P. Friess, P. Guillemin, S. Gusmeroli, et al., "Internet of Things Strategic Research Agenda", Chapter 2 in Internet of Things - Global Technological and Societal Trends, River Publishers, 2011, ISBN 978-8792329-67-7.

[4]. O. Vermesan, R. Bahr, A. Gluhak, F. Boesenberg, A. Hoeer and M. Osella, "IoT Business Models Framework", 2016, online at http://www. internet-of-thingsresearch.eu/pdf/D02 01 WP02 H2020 UNIFYIoT Final.pdf

[5]. A. Gluhak, O. Vermesan, R. Bahr, F. Clari, T. Macchia, M. T. Delgado, A. Hoeer, F. Boesenberg, M. Senigalliesi and V. Barchetti, "Report on IoT platform activities", 2016, online at http://www.internet-of-thingsresearch.eu/pdf/D03 01 WP03 H2020 UNIFYIoT Final.pdf.

[6]. The Internet of Robotic Things, Bioresearch, AN-1818, online at https://www.abiresearch.com/market-

research/product/1019712-the- internet-ofrobotic-things/

[7]. J. Wan, S. Tang, H. Yan, D. Li, S. Wang, and A. V. Vasilakos, "Cloud robotics: Current status and open issues," IEEE Access, vol. 4, pp. 27972807, 2016.

[8]. L. Riazuelo et al., "RoboEarth semantic mapping: A cloud enabled knowledge-based approach," IEEE Trans. Autom. Sci. Eng., vol. 12, no. 2, pp. 432-443, Apr. 2015.

[9]. G. Hu, W. P. Tay, and Y. Wen, "Cloud robotics: Architecture, challenges and applications,"
IEEE Netw., vol. 26, no. 3, pp. 21-28, May/Jun. 2012.

[10]. IEEE Society of Robotics and Automation's Technical Committee on Networked Robots. online at http://www.ieee-ras.org/technicalcommit tees/117-technicalcommittees/networked-robots/146-networkedrobots-Outlier Ventures Research.

[11]. M. Luko`sevic'ius and H. Jaeger. "Reservoir computing approaches to recurrent neural network training." Computer Science Review, vol. 3(3), pp. 127-149, 2009.

[12]. D. Bacciu, P. Barsocchi, S. Chessa, C. Gallicchio, A. Micheli, "An experimental characterization of reservoir computing in ambient assisted living applications", Neural Computing and Applications, vol. 24(6), pp. 1451-1464, 2014.

[13]. M. Luko`sevic'ius, H. Jaeger, B. Schrauwen, "Reservoir Computing Trends", KI $\mathrm{Ku}$ “nstliche Intelligenz, vol. 26(4), pp. 365-371, 2012.

[14]. M. Dragone, G. Amato, D. Bacciu, S. Chessa, S. Coleman, M. Di Rocco, C. Gallicchio, C. Gennaro, H. Lozano, L. Maguire, M. McGinnity, A. Micheli, G. M.P. O’Hare, A. Renteria, A. Saffiotti, C. Vairo, P. Vance, "A Cognitive Robotic Ecology Approach to SelfConfiguring and Evol- ving AAL Systems", Engineering Applications of Artificial Intelligence, Elsevier, vol. 45, pp. 269-280, 2015.

[15]. G.S. Sukhatme, and Mataric', M.J., Embedding Robots Into the Inter- net, Communications of the ACM, 43(5) special issue on Embedding the Internet, D. Estrin, R. Govindan, and J. Heidemann, eds. May, pp. 67-73, 2000

[16]. A. B., Craig, Understanding Augmented Reality - Concepts and Applications, 1st Edition, ISBN: 9780240824086, Morgan Kaufmann, 2013.

[17]. M. Gianni, F. Ferri and F. Pirri ARE: Augmented Reality Environment for Mobile 
Robots. In: A. Natraj, S. Cameron, C. Melhuish, and M. Witkowski (eds) Towards Autonomous Robotic Systems. TAROS 2013. Lecture Notes in Computer Science, vol. 8069, Springer, Berlin, Heidelberg, 2014.

[18]. B.-K. Shim, K.-W. Kang, W-S. Lee2, J-B. Won, and S-H. Han, An Intelligent Control of Mobile Robot Based on Voice Command, 2012 12th International Conference on Control, Automation and Systems, Oct. 17-21, 2012 in ICC, Jeju Island, Korea, 2012.

[19]. IEEE RAS Technical Committee on Networked Robots, 6/10/2017, online at http://networkedrobots.cs.umn.edu/

[20]. Bit coin: Beyond money, Deloitte University Press, DUPress.com, online at https://dupress.deloitte.com/content/dam/dupus-en/articles/bitcoin- fact-fiction-future/DUP 847BitcoinFactFictionFuture.pdf

[21]. E. C. Ferrer, The block chain: a new framework for robotic swarm systems, Cornell University Library, August 2016, online at https://arxiv.org/pdf/1608.00695.pdf.

[22]. Kamakshaiah Kolli etc... A Traditional Analysis for Efficient Data Mining with Integrated Association Mining into Regression Techniques, 3rd International Conference on Communications and Cyber Physical Engineering, LNEE, volume 698,pp 1393-1404, ISBN: 978-981-15-7961-5.

[23]. Syed Umar et el., "Securities and threats of cloud computing and solutions" 2018 2nd International Conference on Inventive Systems and Control (ICISC), IEEE Explorer, Pages: 1162-1166.

\section{Cite this article as :}

Syed Umar, Endal Kachew Mosisa, "A Survey on Evolution of Cognitive Robotics with Internet of Things", International Journal of Scientific Research in Science, Engineering and Technology (IJSRSET), Online ISSN : 2394-4099, Print ISSN : 2395-1990, Volume 8 Issue 2, pp. 337-344, March-April 2021. Available at doi : https://doi.org/10.32628/IJSRSET218254 Journal URL : https://ijsrset.com/IJSRSET218254 